\title{
La farmacia comunitaria como fuente de información de la calidad del aire de las ciudades. A propósito de un estudio de opinión
}

\author{
Juan Carlos López-Gil ${ }^{1,4}$, María del Carmen Carreter-Garnica ${ }^{2,4}$, José María Ordóñez-Iriarte ${ }^{3,4}$ \\ 1. Graduado en Farmacia. 2. Graduada en Farmacia. 3. Doctor en Salud Pública. 4. Departamento de Farmacia, Facultad de Ciencias Experimentales, \\ Universidad Francisco de Vitoria (UFV), Madrid.
}

\section{PALABRAS CLAVE}

Contaminación del aire, servicios de farmacia comunitaria, encuestas epidemiológicas, salud pública

\section{RESUMEN}

Introducción: la Comunidad de Madrid soporta episodios de alta contaminación que le han obligado a elaborar protocolos de actuación que incluyen informar a los ciudadanos. La farmacia comunitaria podría informar al paciente, no sólo de los riesgos de la exposición a los contaminantes, sino también de pautas de prevención ante ellos. El objetivo de este estudio ha sido conocer si la población sabe si la contaminación afecta a su salud y si la farmacia puede ser un buen canal para difundir dicha información.

Materiales y métodos: estudio epidemiológico observacional de tipo transversal $(\mathrm{n}=284)$. La recogida de la información se hizo mediante entrevista personal, realizada por un farmacéutico en las farmacias colaboradoras, desde enero a junio de 2018.

Resultados: el 94,2 \% de los encuestados cree que la contaminación atmosférica tiene implicaciones en la salud y conoce su riesgo. Un 45,4 \% refiere no saber cuándo se superan los niveles de contaminación en el municipio donde vive. Solo un 14,1 \% recibía información por los canales de la Administración. El 86,9 \% cree no recibir suficiente información en materia de contaminación y salud. Al 74,6 \% le gustaría recibir dicha información y un 68,8 \% [IC (95 \%): 63,0-74,5], la querría de forma individual a través de la farmacia comunitaria.

Discusión: si bien la población sabe que la contaminación atmosférica afecta a su salud, no recibe suficiente información. Los sistemas empleados por la Administración no son efectivos y se identifica a la farmacia comunitaria como un lugar idóneo para recibir la información requerida.

Community pharmacy as an information source about the air quality of cities. About an opinion study

\section{ABSTRACT}

Introduction: Madrid region suffers high pollution level episodes, leading it the elaboration of action protocols that include informing citizens. From the community pharmacy, we could seek to inform the patient, not only of the risks of exposure to this scenario, but also about prevention guidelines against it. The objective of this study was to know if there is a need of information about how contamination affects health and if the pharmacy can be a good channel to disseminate this information.

Materials and Methods: Observational epidemiological transversal study $(\mathrm{n}=284)$. The information was collected through a personal interview conducted by a pharmacist in the collaborating pharmacies from January to June 2018.

Results: $94.2 \%$ of respondents believe that air pollution has health implications and knows its risks. 45.4\% report not knowing when pollution levels are exceeded in the town where they live. Only 14,1\% received information through the administration channels. $86.9 \%$ believe that they do not receive enough information regarding pollution and health. 74.6\% wants to receive this information and 68.8\% [CI (95\%): 63,0-74,5], want it individually and using the community pharmacy as a channel.

Discussion: Citizens know that air pollution affects their health, although they do not receive enough information. The systems used by the administration are not effective and that postulates the community pharmacy as an agent of interest for the distribution of the required information.

Una exposición preliminar de estos resultados fue expuesta en forma de póster en el VIII Congreso Nacional de Farmacéuticos Comunitarios de Alicante en 2018.

Recibido: 10/7/2019

Aceptado: 17/12/2019

Disponible online: 6/3/2020
Financiación: ninguna.

Conflicto de intereses: ninguno.

Cite este artículo como: López-Gil JC, Carreter-Garnica MC, Ordóñez-Iriarte JM. La farmacia comunitaria como fuente de información de la calidad del aire de las ciudades. A propósito de un estudio de opinión. Farmacéuticos Comunitarios. 2020 Mar 06:12(1):5-16. doi:10.5672/FC.2173-9218.(2020/Vol12).001.02

Correspondencia: Juan Carlos López-Gil (juancarlosbio95@gmail.com).

ISSN 1885-8619 OSEFAC (Sociedad Española de Farmacia Familiar y Comunitaria). Todos los derechos reservados. 


\section{Introducción}

Si bien es cierto que siempre ha habido contaminación del aire, la llamada Revolución Industrial supuso un punto de inflexión en la misma. Sin embargo, hubo que esperar a los años 30 y al final de la Segunda Guerra Mundial para conocer el alcance de este fenómeno. Sirva de ejemplo para ello, los clásicos episodios del Valle del Mosa (Bélgica, 1930), Donora (Pensilvania, 1948) o Londres (1952), saldados cada uno de ellos con un número relevante de fallecidos (1).

En las ciudades españolas se ha producido una transición desde la década de los años 70. El "humo negro" y el $\mathrm{SO}_{2}$ debidos a las calefacciones eran los contaminantes más importantes, pero a partir de los años 90 la situación se redefinió de forma que las partículas, medidas como partículas menores de 10 y 2,5 micras (PM10 y PM2.5, respectivamente), y el $\mathrm{NO}_{2}$, originados fundamentalmente por el tráfico rodado, son los que vienen dibujando el panorama actual de la calidad del aire (2).

La evidencia de que los niveles actuales de contaminación del aire de nuestras ciudades pueden perjudicar la salud son cada vez mayores (3). Estudios multicéntricos europeos como el Air pollution and health: A European approach (APHEA), Air Pollution and Health- A European Information System (APHEIS), el estudio americano National Morbidity, Mortality and Air Pollution Study (NMMAPS) o los españoles Estudio Multicéntrico Español entre la Contaminación Atmosférica y Mortalidad (EMECAM) y Estudio Multicéntrico en España de los Efectos a corto plazo de la Contaminación Atmosférica sobre la Salud (EMECAS), así lo han establecido (2). Un reciente estudio ha recogido los siguientes datos del impacto de las PM2.5: fueron las causantes del 5,3\% del total de la mortalidad global, lo que las convirtió en el séptimo factor de riesgo de mortalidad; causaron 2,9 millones de muertes y 69,7 millones de años de vida perdidos (4).

El proyecto EMECAS, en consonancia con los otros estudios multicéntricos citados, encontró que el aumento de estos contaminantes se asociaba con el incremento de ingresos hospitalarios por enfermedades cardiovasculares y respiratorias, siendo las más afectadas el asma, la EPOC y el infarto de miocardio (5).

En el caso de la EPOC, las PM2.5 inducen estrés oxidativo a nivel pulmonar y propician un ambiente proinflamatorio con efectos en el sistema inmune, la microbiota del tracto respiratorio o el sistema cardiovascular (4). Las PM10, por su parte, se asocian significativamente con un aumento de exacerbaciones agudas y de mortalidad por EPOC cuando hay un aumento de $10 \mu \mathrm{g} / \mathrm{m}^{3}$ (4).

$\mathrm{Al}$ igual que en la EPOC, el asma viene caracterizado por una inflamación de las vías aéreas. Las PM2.5 y las PM10 han mostrado efectos a largo y corto plazo sobre los pacientes con asma. La pérdida de capacidad pulmonar debido a estos compuestos da lugar a un asma mal controlado y con mayor riesgo de crisis (6). En la Comunidad de Madrid se vio que un incremento de $1 \mathrm{mg} / \mathrm{m}^{3}$ de la máxima horaria de $\mathrm{CO}$, se asociaba con un incremento de 2,6\% de los ingresos hospitalarios por asma (5).

En relación a las enfermedades cardiovasculares los resultados obtenidos han sido diversos. En Bélgica se ha correlacionado la exposición a un aumento de $10 \mu \mathrm{g} / \mathrm{m}^{3}$ en PM2.5 y en $\mathrm{NO}_{2}$ sobre el nivel basal, con un incremento en el riesgo de padecer un tipo específico de infarto de miocardio (7). En la Comunidad de Madrid esos mismos resultados se han visto con incrementos de $1 \mathrm{mg} / \mathrm{m}^{3}$ de la máxima media octohoraria diaria de CO del mismo día, y con un aumento del 2,1 \% de ingresos por enfermedad isquémica cardiaca. Con el ozono también se encontró relación significativa con un aumento del 1,1 \% de ingresos (5).

Además, la International Agency for Research on Cancer (IARC) ha clasificado la contaminación atmosférica como un agente carcinógeno en humanos (Grupo 1) (8).

La ciudad de Madrid y los municipios periurbanos, como muchas ciudades europeas de similares características, se enfrentan al problema del $\mathrm{NO}_{2}$ derivado del tráfico rodado. Para abordarlo, se publicó el Decreto $140 / 2017$, de 21 de noviembre, por el que se aprueba el protocolo para los episodios de alta contaminación (9), texto que se redactó después del aprobado por el Ayuntamiento de Madrid en 2016 (10).
Por otro lado, se asiste a un nuevo modelo de Farmacia, entendida como un servicio asistencial, que viene avalado por el Consenso sobre Atención Farmacéutica de 2001 (11), donde se reclama a las instituciones la posibilidad de aumentar el marco de actuación del farmacéutico. Se busca respetar y ejecutar las tres actividades básicas de la Atención Farmacéutica, así como otras que complementen y enriquezcan los servicios prestados. Además, el Proyecto de Ley de Farmacia de la Comunidad de Madrid recoge esta vocación asistencial, dejando abierta la intervención de la Farmacia Comunitaria y Hospitalaria en materia de Salud Pública y Sanidad Ambiental, tal como se recoge en el artículo 15, apartado 2a. Recientemente, también el Consejo General de Colegios Oficiales de Farmacéuticos (CGCOF) ha aprobado el Código Deontológico de la profesión, que en el artículo 33 define la intervención del farmacéutico en programas que puedan detectar y corregir problemas potenciales de salud en la población, además de incentivar la colaboración con las Administraciones sanitarias en materia de salud pública (12).

Dado la trascendencia de la contaminación atmosférica por el efecto que ésta tiene sobre determinadas enfermedades, se puede entender como un problema de salud pública que podría tenerse en cuenta a la hora de elaborar los protocolos de Atención Farmacéutica de determinadas enfermedades, estableciendo otra vía de información para el paciente y enriqueciéndola con el consejo farmacéutico.

El objetivo de este estudio es determinar si existe necesidad de información por parte de los madrileños sobre cómo la contaminación afecta a su salud y si la farmacia puede ser un buen canal para difundir dicha información.

\section{Materiales y métodos}

\section{Diseño del estudio}

Estudio epidemiológico observacional de tipo transversal llevado a cabo entre febrero y junio de 2018. El tamaño muestral se calculó en función de la población mayor de edad de la Comunidad de Madrid, según el último padrón (2017) para una confianza del $95 \%$, una precisión del $5 \%$ y una frecuencia observada del $75 \%$. 
Se obtuvo con EpiInfo ${ }^{\circledR}$ una muestra necesaria de 227 individuos a los que encuestar. Se reclutaron 284 usuarios en 20 farmacias comunitarias de la Comunidad de Madrid entre todos los usuarios mayores de edad que acudieron a las mismas, vivían en esta Comunidad y quisieron participar.

Recogida de la información y análisis estadístico

Se diseñó un cuestionario propio en Google Forms ${ }^{\circledR}$ (anexo) que fue cumplimentado por los farmacéuticos de las 20 farmacias comunitarias con los datos de los participantes. Dada la distribución zonal de la Comunidad de Madrid en 7 áreas en cuanto a calidad del aire (Madrid, corredor del Henares, urbana Sur, urbana Noroeste, Sierra Norte, Cuenca del Alberche y Cuenca del Tajuña), se priorizó obtener datos del área metropolitana, alrededores y las zonas próximas a núcleos urbanos con una población superior a 50.000 habitantes, dado que son las que sufren de manera insidiosa los efectos de la contaminación. Se recogieron entre 10 y 15 encuestas por cada farmacia. Los datos obtenidos se almacenaron en una base de datos y fueron procesados con EpiInfo ${ }^{\circledR}$, versión 7.2.2.6. Se realizaron transformaciones de la variable "Estudios" para llevar a cabo algunos análisis. En concreto se recodificó a dos categorías: "Estudios básicos", que englobó a "Sin graduado escolar", "Primaria completa" y "ESO", y "Estudios superiores" que recogió a las otras dos variables. Para el análisis estadístico se utilizaron las tablas de contingencia y la prueba de la $\chi^{2}$; en aquellas ocasiones en que fueron apropiadas, se recurrió a pruebas no paramétricas como la correlación de Spearman.

\section{Asignación de la exposición}

Se asignó al centroide de cada código postal de residencia recogido en el formulario los valores mínimos anuales de $\mathrm{NO}_{2}$ según las mediciones realizadas por el Sistema Integral de Vigilancia, Predicción e Información de la Calidad del Aire del Ayuntamiento de Madrid (13) y por la Red de Calidad del Aire de la Comunidad de Madrid (14). Así se pudo categorizar la exposición a la que se ven sometidos los pacientes en su vivienda. Se usaron los datos del año 2017 por ser especialmente insidioso en cuanto a contaminación atmosférica por $\mathrm{NO}_{2}$ $\mathrm{y}$, por tanto, establecer un escenario poco favorable para la calidad del aire. La asignación de la exposición en la vivienda de cada participante se hizo en función del valor de las isolíneas de concentración: mayores, menores o iguales a $40 \mu \mathrm{g} / \mathrm{m}^{3}$, que es el valor medio diario anual permitido para este contaminante según la legislación vigente. Esto diferenció la residencia en zona de exposición alta, baja o límite.

\section{Consideraciones éticas}

La participación voluntaria llevaba implícito el consentimiento informado. Se respetó la normativa de protección de datos. Se contó con la autorización del Comité de Ética del Comité de Investigación de la Universidad Francisco de Vitoria.

\section{Resultados}

La muestra final fue de 260 personas (de las 284 reclutadas) porque solo se consideraron a los que vivían en la Comunidad de Madrid (tabla 1). En la figura 1, donde están las isolíneas de concentración de $\mathrm{NO}_{2}$, se dibujan los códigos postales donde viven los participantes en este estudio.

Se ha encuestado a un total de 173 mujeres y 87 hombres, de los cuales más de las tres cuartas partes se encuentran distribuidos entre los dos primeros grupos de edad (18-40 y 4165), mientras que el grupo de mayores de 65 años supone el 18,9 \% del total; esta variable muestra significación estadística al segregar por nivel de contaminación por $\mathrm{NO}_{2}$, en el domicilio, en el sentido de que las personas de mayor edad viven en zonas más contaminadas. El 47,5 \% de la población encuestada tiene título universitario, siendo el nivel de estudios mayoritario en contraposición del no tener estudios, que corresponde al 7,7 \% de los encuestados. La $r$ de Spearman, que es significativa, señala la correlación pequeña, pero inversa, entre nivel de estudios y residir en zonas contaminadas. La mayoría (54,2 \%) no padecía ningún tipo de enfermedad de las contempladas en el estudio, que son enfermedades que se verían agravadas ante una mayor concentración de contaminantes en el aire.
De la muestra, las referidas mayoritariamente fueron la alergia al polen $(23,8 \%)$ y la hipertensión (18,5\%).

En la tabla 2 se recoge la información sobre la percepción del riesgo. El 94,2 \% de los encuestados respondió que la contaminación tiene implicaciones en la salud, y esos porcentajes se mantienen sin apenas variación cuando se pregunta si la contaminación en su municipio le afecta a su salud. Los grupos de población que se verían más afectados, según la opinión de los encuestados (solo se recogen los datos afirmativos y la respuesta ha sido múltiple) serían las personas con enfermedades respiratorias (90,4\%), los ancianos $(78,1 \%)$ y los niños (70 \%); por su parte, embarazadas con un 45,4 \% y adultos sanos con el 17,7 \%, fueron los que menos. Ninguna de estas variables, analizadas según área de contaminación en la que vivían, resultó significativa desde el punto de vista estadístico.

$\mathrm{Al}$ analizar los resultados obtenidos según las variables sociodemográficas planteadas (tabla 2), no se han encontrado diferencias significativas en la percepción del riesgo de la contaminación atmosférica ni por la variable del sexo ni por edad. Sin embargo, la variable de estudios sí que muestra diferencias significativas ( $p=0,03$ ), en el sentido de que los que tiene más estudios tiene más conciencia de este riesgo, aunque vivan en áreas menos contaminadas.

El 54,6 \% de la población respondió que sí conocía cuándo se superaban los límites legislados para la calidad del aire, mientras que un $45,4 \%$ lo desconocía, sin diferencias estadísticamente significativas según la zona de residencia (tabla 3).

La población que afirmaba ser conocedora de esta información, mayoritariamente la recibe a través de los medios de comunicación como la televisión o la radio (71,1\%). El teléfono móvil y las comunicaciones institucionales (carteles en carreteras, anuncios por páginas web oficiales, avisos a través del Ayuntamiento...) quedan como las fuentes menos empleadas: un 14,8 \% y un $14,1 \%$, respectivamente. Además, la mayoría de la población encuestada $(57,3 \%)$ reside en la ciudad de Madrid, que es la principal afectada por estos episodios de alta contaminación (figura 1). 
Tabla 1 Datos sociodemográficos de la muestra en relación a los niveles de $\mathrm{NO}_{2^{\prime}}$ en el área domiciliaria y enfermedades más frecuentes

\begin{tabular}{|c|c|c|c|c|c|}
\hline \multirow{2}{*}{\multicolumn{2}{|c|}{ Variable }} & \multicolumn{3}{|c|}{ Nivel de $\mathrm{NO}_{2}$, en el área domiciliaria* } & \multirow[b]{2}{*}{$\mathrm{p}$} \\
\hline & & Alta [n; $(\%)]$ & Límite [n (\%)] & Baja [n (\%)] & \\
\hline \multicolumn{2}{|l|}{ Total } & $73(28,1)$ & $70(26,9)$ & $117(45)$ & \\
\hline \multicolumn{6}{|l|}{$\operatorname{Sexo}[n ;(\%)]$} \\
\hline \multicolumn{2}{|l|}{ Mujeres: $173(66,5)$} & $43(58,9)$ & $45(64,3)$ & $85(72,7)$ & \multirow{2}{*}{ NS } \\
\hline \multicolumn{2}{|l|}{ Hombres: $87(33,5)$} & $30(41,1)$ & $25(35,7)$ & $32(27,4)$ & \\
\hline \multicolumn{6}{|l|}{ Edad $[n ;(\%)]$} \\
\hline \multicolumn{2}{|l|}{ 18-40: $105(40,4)$} & $33(45,2)$ & $22(31,4)$ & $50(42,7)$ & \multirow{3}{*}{0,03} \\
\hline \multicolumn{2}{|l|}{ 41-65: $106(40,8)$} & $22(30,1)$ & $31(44,3)$ & $53(45,3)$ & \\
\hline \multicolumn{2}{|l|}{$>65: 49(18,8)$} & $18(24,7)$ & $17(24,3)$ & $14(12,0)$ & \\
\hline \multicolumn{6}{|l|}{ Estudios $[n ;(\%)]$} \\
\hline \multicolumn{2}{|c|}{ Sin graduado escolar: $22(8,5)$} & $7(9,6)$ & $11(15,7)$ & $4(3,42)$ & \multirow{5}{*}{ NS } \\
\hline \multicolumn{2}{|c|}{ Primaria completa: $32(12,3)$} & $10(13,7)$ & $11(15,7)$ & $11(9,4)$ & \\
\hline \multicolumn{2}{|c|}{ ESO: $32(12,3)$} & $10(13,7)$ & $10(14,3)$ & $12(10,3)$ & \\
\hline \multicolumn{2}{|c|}{ Bachillerato: $39(15,0)$} & $9(12,3)$ & $10(14,3)$ & $20(17,9)$ & \\
\hline \multicolumn{2}{|c|}{ Estudios universitarios: $135(51,9)$} & $37(50,7)$ & $28(40,0)$ & $70(59,8)$ & \\
\hline 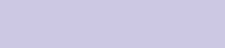 & 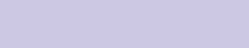 & & & $r^{* *}=-0,152$ & 0,014 \\
\hline \multicolumn{6}{|c|}{ Estudios recodificados $[n ;(\%)]$} \\
\hline \multicolumn{2}{|c|}{ Estudios básicos: $86(33,1)$} & $27(37,0)$ & $32(45,7)$ & $27(23,1)$ & \multirow{2}{*}{0,004} \\
\hline Estudios superiore & : $174(66,9)$ & $46(63,0)$ & $38(54,3)$ & $90(76,9)$ & \\
\hline \multicolumn{6}{|l|}{ Enfermedades } \\
\hline \multicolumn{2}{|l|}{ Si (Total): 45,8 \% } & $40(54,8)$ & $37(52,9)$ & $64(54,7)$ & \multirow{2}{*}{ NS } \\
\hline \multicolumn{2}{|l|}{ No (Total): $54,2 \%$} & $33(45,2)$ & $33(47,1)$ & $53(45,3)$ & \\
\hline Alergia al polen & $\begin{array}{l}\text { Si (Total: } 23,8 \% \text { ) } \\
\text { No (Total: } 76,2 \% \text { ) }\end{array}$ & $\begin{array}{l}14(19,2) \\
59(80,8)\end{array}$ & $\begin{array}{l}14(20,0) \\
56(80,0)\end{array}$ & $\begin{array}{l}34(29,1) \\
83(70,9)\end{array}$ & NS \\
\hline Asma & $\begin{array}{l}\text { Si (Total: } 13,5 \% \text { ) } \\
\text { No (Total: } 86,5 \% \text { ) }\end{array}$ & $\begin{array}{c}6(8,2) \\
67(91,8)\end{array}$ & $\begin{array}{l}10(14,3) \\
60(85,7)\end{array}$ & $\begin{array}{l}19(16,2) \\
98(83,8)\end{array}$ & NS \\
\hline Bronquitis o EPOC & $\begin{array}{l}\text { Si (Total: 6,2 \%) } \\
\text { No (Total: 93,8 \%) }\end{array}$ & $\begin{array}{c}5(6,9) \\
68(93,1)\end{array}$ & $\begin{array}{c}6(5,1) \\
64(94,9)\end{array}$ & $\begin{array}{c}5(7,1) \\
112(92,9)\end{array}$ & NS \\
\hline $\begin{array}{l}\text { Enfermedades } \\
\text { cardiacas }\end{array}$ & $\begin{array}{l}\text { Si (Total: 9,2 \%) } \\
\text { No (Total: 90,8 \%) }\end{array}$ & $\begin{array}{c}6(8,2) \\
67(91,8)\end{array}$ & $\begin{array}{c}8(11,4) \\
62(88,6)\end{array}$ & $\begin{array}{c}10(8,6) \\
107(91,4)\end{array}$ & NS \\
\hline Hipertensión & $\begin{array}{l}\text { Si (Total: } 18,5 \%) \\
\text { No (Total: } 81,5 \% \text { ) }\end{array}$ & $\begin{array}{l}13(17,8) \\
60(82,2)\end{array}$ & $\begin{array}{l}16(22,9) \\
54(77,1)\end{array}$ & $\begin{array}{c}19(16,2) \\
(83,8)\end{array}$ & NS \\
\hline
\end{tabular}

${ }^{*}$ Alta: $>40$; limite $=40 ;$ Baja $<40 \mu \mathrm{g} / \mathrm{m}^{3}$. NS: no significativo; $\mathrm{r}^{* *}=\mathrm{r}$ de Spearman.

Por otro lado, la mayoría de la población encuestada responde que no recibe suficiente información acerca de cómo la contaminación afecta a la salud (86,9 \%), excediendo el $80 \%$ en las tres áreas establecidas, sin encontrarse diferencias estadísticamente significativas entre ellas.

El 74,6 \% de los encuestados refirieron querer recibir información en materia de contaminación y salud y el 51,9\% prefería que esta fuera de forma colectiva (medios de comunicación, pantallas en las ciudades...), tal como se está haciendo actualmente.

En relación a la farmacia comunitaria como el canal de informa- ción sobre calidad del aire, el 68,8\% (IC95 \%: 63,0-74,5 \%) de los encuestados valora positivamente que este establecimiento fuese quien les informase individualmente en materia de contaminación atmosférica y salud. Este tipo de información sería ofrecida de manera individualizada en la farmacia comunitaria. Se encuentran diferencias estadísticamente significativas ( $p=0,038$ ), siendo las personas que viven en zonas menos contaminadas las que muestran mayor interés porque la farmacia sea fuente de información (tabla 4).

Al analizar estos datos, se ve que no existen diferencias por sexo ni por estudios; sin embargo, sí existen diferencias significativas desde el punto de vista estadístico respecto a la edad, en el sentido de que, a menor edad y menor nivel de contaminación en el domicilio, mayor interés en recibir información desde la farmacia comunitaria. Analizando la variable nivel de estudio, tanto en forma no paramétrica ( $\mathrm{r}$ de Spearman) como reagrupándola en dos, resulta estadísticamente significativa en cuanto a que, a mayores estudios, mayor es el interés en considerar la farmacia comunitaria como buen lugar para recibir información sobre calidad del aire y salud. 


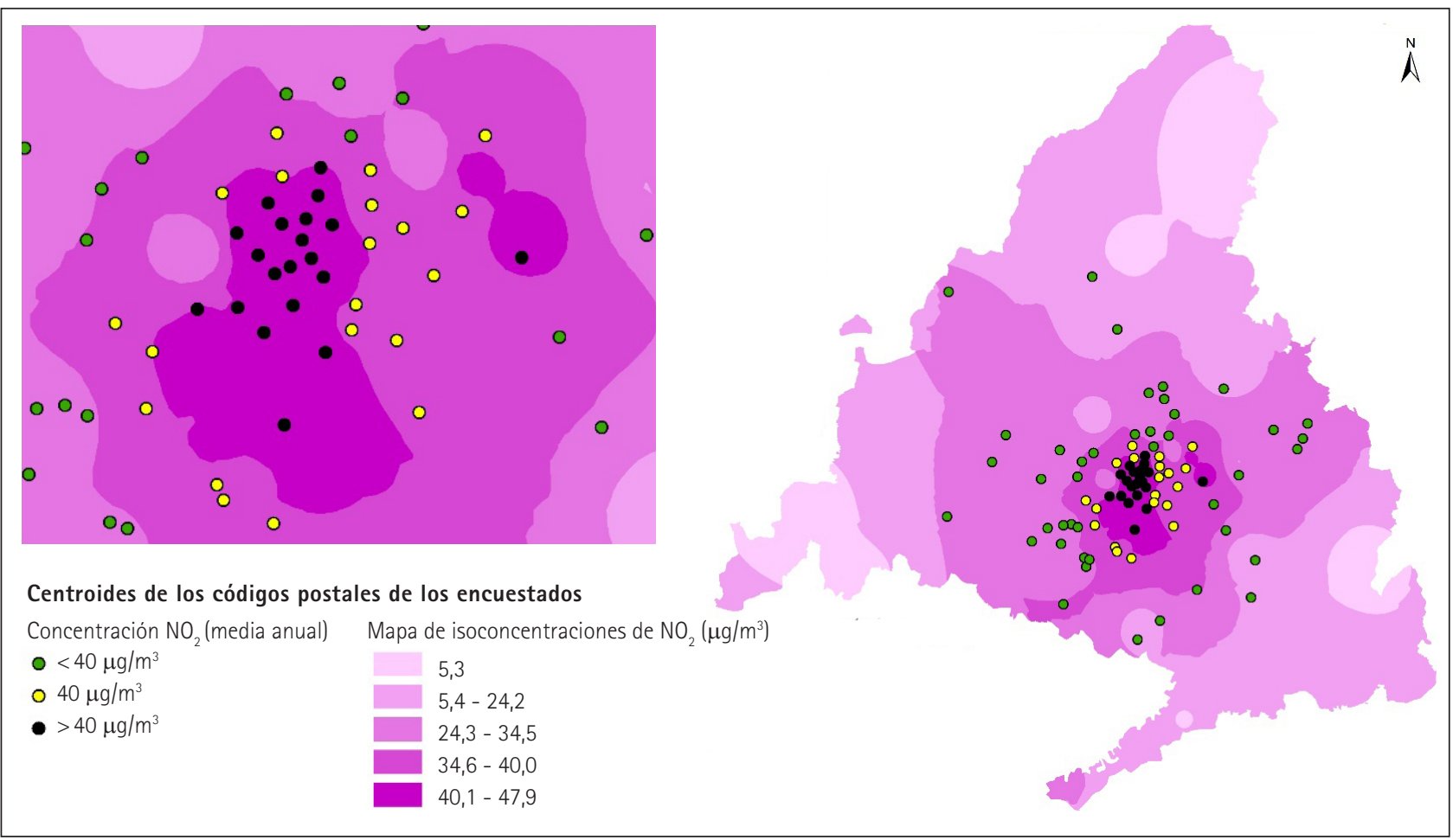

Figura 1 Centroides de los códigos postales de los encuestados en relación a las isolíneas de concentración que definen las tres áreas de contaminación

Tabla 2 Percepción de la relación entre contaminación y salud en relación a los niveles de $\mathrm{NO}_{2^{\prime}}$ en el área domiciliaria

\begin{tabular}{|c|c|c|c|c|}
\hline \multirow{2}{*}{ Pregunta y opciones } & \multicolumn{3}{|c|}{ Nivel de $\mathrm{NO}_{2}$, en el área domiciliaria } & \multirow{2}{*}{ p } \\
\hline & Alta $[n(\%)]$ & Límite [n (\%)] & Baja [n (\%)] & \\
\hline \multicolumn{5}{|c|}{ ¿Cree Ud. que la contaminación tiene implicaciones en su salud? } \\
\hline Si (Total: $94,2 \%)$ & $70(95,9)$ & $66(94,3)$ & $109(93,2)$ & \multirow[b]{2}{*}{ NS } \\
\hline No (Total: 5,8 \%) & $3(4,1)$ & $4(5,7)$ & $8(6,8)$ & \\
\hline \multicolumn{5}{|c|}{ ¿Cree Ud. que la contaminación del municipio donde vive tiene implicaciones en su salud? } \\
\hline Si (Total: 93,1\%) & $66(90,4)$ & $67(95,7)$ & $109(93,2)$ & \multirow[b]{2}{*}{ NS } \\
\hline No (Total: 6,9\%) & $7(9,6)$ & $3(4,3)$ & $8(6,8)$ & \\
\hline \multicolumn{5}{|c|}{ Entre los que sí creen que la contaminación tiene implicaciones en salud } \\
\hline \multicolumn{5}{|l|}{ Sexo } \\
\hline Mujeres & $41(58,6)$ & $42(63,6)$ & $78(71,6)$ & \multirow{2}{*}{ NS } \\
\hline Hombres & $29(41,4)$ & $24(36,4)$ & $31(28,4)$ & \\
\hline \multicolumn{5}{|l|}{ Edad } \\
\hline $18-40$ & $32(45,7)$ & $22(33,3)$ & $48(44,0)$ & \multirow{3}{*}{ NS } \\
\hline $41-65$ & $22(31,4)$ & $31(47,0)$ & $48(44,0)$ & \\
\hline$>65$ & $16(22,9)$ & $13(19,7)$ & $13(12,0)$ & \\
\hline \multicolumn{5}{|l|}{ Estudios recodificados } \\
\hline Estudios básicos & $24(34,3)$ & $28(42,4)$ & $26(23,9)$ & \multirow{2}{*}{0,03} \\
\hline Estudios superiores & $46(65,7)$ & $38(57,6)$ & $83(76,1)$ & \\
\hline \multicolumn{5}{|c|}{ ¿Qué grupos de personas son los más afectados por la contaminación? ${ }^{* *}$} \\
\hline Adultos sanos $\quad$ (Total: $17,7 \%$ ) & $9(12,3)$ & $14(20,0)$ & $23(19,7)$ & \\
\hline (Total: 78,1 \%) & $63(86,3)$ & $51(72,9)$ & $89(76,1)$ & \\
\hline Enfermedades respiratorias (Total: 90,4\%) & $65(89,0)$ & $66(94,3)$ & $117(88,9)$ & \\
\hline Enfermedades cardiacas $\quad$ (Total: $42,7 \%$ ) & $32(43,8)$ & $30(42,9)$ & $49(41,9)$ & \\
\hline (Total: 45,4 \%) & $29(39,7)$ & $34(48,6)$ & $55(47,0)$ & \\
\hline (Total: $70 \%$ ) & $54(74,0)$ & $47(67,1)$ & $81(69,2)$ & NS \\
\hline
\end{tabular}

${ }^{*}$ Alta: $>40$; limite $=40 ;$ Baja $<40 \mu \mathrm{g} / \mathrm{m}^{3}$. NS: no significativo. ${ }^{* *}$ Respuesta múltiple. 
Tabla 3 Conocimiento de las superaciones de los niveles de $\mathrm{NO}_{2}$, en el área domiciliaria de los encuestados en la Comunidad de Madrid y percepción sobre la información que reciben

\begin{tabular}{|c|c|c|c|c|}
\hline \multirow[b]{2}{*}{ Pregunta y opciones } & \multicolumn{3}{|c|}{ Nivel de $\mathrm{NO}_{2}$ en el área domiciliaria* } & \multirow[b]{2}{*}{$\mathrm{p}$} \\
\hline & Alta [n (\%)] & Límite [n (\%)] & Baja [n (\%)] & \\
\hline \multicolumn{5}{|c|}{ ¿Sabe cuándo se superan los niveles de contaminación en su municipio? } \\
\hline Si (Total: $54,6 \%)$ & $42(57,5)$ & $42(60,0)$ & $58(49,6)$ & \multirow[b]{2}{*}{ NS } \\
\hline No (Total: 45,4\%) & $31(42,5)$ & $28(40,0)$ & $59(50,4)$ & \\
\hline \multicolumn{5}{|c|}{ En caso afirmativo, ¿por qué medio le llega la información? } \\
\hline A través del móvil $\quad$ Si (Total: $14,7 \%)$ & $5(11,9)$ & $5(11,9)$ & $11(19,0)$ & \multirow[b]{3}{*}{ NS } \\
\hline Medios de comunicación & $29(69,0)$ & $33(78,6)$ & $39(67,2)$ & \\
\hline Comunicación institucional Si (Total: 14,1\%) & $8(19,1)$ & $4(9,5)$ & $8(13,8)$ & \\
\hline \multicolumn{5}{|c|}{ ¿Cree que recibe suficiente información sobre cómo la contaminación afecta a la salud? } \\
\hline Si (Total: $13,1 \%)$ & $11(15,1)$ & $8(11,4)$ & $15(12,8)$ & \multirow[b]{2}{*}{ NS } \\
\hline No (Total: (86,9 \%) & $62(84,9)$ & $62(88,6)$ & $102(87,2)$ & \\
\hline \multicolumn{5}{|c|}{ ¿Le gustaría recibir información sobre cómo la contaminación afecta a su salud? } \\
\hline Si (Total: $74,6 \%)$ & $48(65,8)$ & $54(77,1)$ & $92(78,6)$ & \multirow[b]{2}{*}{ NS } \\
\hline No (Total: $25,4 \%)$ & $25(34,2)$ & $16(22,9)$ & $25(21,4)$ & \\
\hline \multicolumn{5}{|c|}{ ¿Le gustaria recibir dicha información de forma individual o colectiva? } \\
\hline Individual (Total: 48,1 \%) & $28(38,4)$ & $33(47,1)$ & $64(54,7)$ & \multirow[b]{2}{*}{ NS } \\
\hline Colectiva (Total: 51,9 \%) & $45(61,6)$ & $37(52,9)$ & $53(45,3)$ & \\
\hline
\end{tabular}

*Alta: > 40; límite = 40; Baja < $40 \mu \mathrm{g} / \mathrm{m}^{3}$. NS: no significativo; En el análisis no se incluyen los "no sabe/no contesta".

Tabla 4 Interés en que sea la farmacia comunitaria la que le informe sobre contaminación y salud en relación a los niveles de $\mathrm{NO}_{2}$ en el área domiciliaria

\begin{tabular}{|c|c|c|c|c|}
\hline \multirow[b]{2}{*}{ Pregunta y opciones } & \multicolumn{3}{|c|}{ Nivel de $\mathrm{NO}_{2}$ en el área domiciliaria* } & \multirow[b]{2}{*}{$\mathrm{p}$} \\
\hline & Alta [n (\%)] & Limite $[n(\%)]$ & Baja [n (\%)] & \\
\hline \multicolumn{5}{|c|}{ ¿Le gustaria que en la farmacia a la que acude le diera dicha información relativa a la contaminación de forma individual? } \\
\hline \multicolumn{5}{|c|}{$\begin{array}{l}\text { No: } 81(31,2) \\
\text { Si: } 179(68,8) ; \text { IC (95\%): } 63,0-74,5)\end{array}$} \\
\hline Si & $45(61,6)$ & $44(62,9)$ & $90(76,9)$ & \multirow{2}{*}{0,038} \\
\hline No & $28(38,4)$ & $26(37,1)$ & $27(23,1)$ & \\
\hline \multicolumn{5}{|l|}{ Sexo } \\
\hline Mujeres: $61(34,1 \%)$ & $17(37,8)$ & $19(43,2)$ & $25(27,8)$ & \multirow{2}{*}{ NS } \\
\hline Hombres: 118 (65,9%) & $28(62,2)$ & $25(56,8)$ & $65(72,2)$ & \\
\hline \multicolumn{5}{|l|}{ Edad } \\
\hline $18-40$ & $22(48,9)$ & $12(27,2)$ & $38(42,2)$ & \multirow{3}{*}{0,037} \\
\hline $45-65$ & $11(24,4)$ & $23(52,2)$ & $39(43,4)$ & \\
\hline$>65$ & $12(26,7)$ & $9(20,6)$ & $13(14,4)$ & \\
\hline \multicolumn{5}{|l|}{ Estudios } \\
\hline Sin graduado escolar & $4(8,9)$ & $8(18,2)$ & $4(4,4)$ & \multirow{5}{*}{ NS } \\
\hline Primaria completa & $8(17,8)$ & $5(11,4)$ & $6(6,7)$ & \\
\hline ESO & $4(8,9)$ & $5(11,4)$ & $8(8,9)$ & \\
\hline Bachillerato & $5(11,1)$ & $6(13,6)$ & $17(18,9)$ & \\
\hline Estudios universitarios & $24(53,3)$ & $20(45,4)$ & $55(61,1)$ & \\
\hline & & & $r^{* *}=-0,175$ & 0,019 \\
\hline \multicolumn{5}{|l|}{ Estudios recodificados } \\
\hline Estudios básicos & $16(35,5)$ & $18(40,9)$ & $18(20,0)$ & \multirow{2}{*}{0,02} \\
\hline Estudios superiores & $29(64,5)$ & $26(59.1)$ & $72(80,0)$ & \\
\hline
\end{tabular}

${ }^{*}$ Alta: $>40$; límite $=40 ;$ Baja $<40 \mu \mathrm{g} / \mathrm{m}^{3}$. NS: no significativo. $r^{* *}=r$ de Spearman. 


\section{Discusión}

La contaminación atmosférica es un factor de riesgo ambiental que suscita una gran preocupación entre los ciudadanos cuando se les pregunta específicamente sobre ella. Así por ejemplo, la Survey of views of stakeholders, experts and citizens on the review on EU Air Policy (15) señala que "más del $80 \%$ de los ciudadanos europeos considera que la calidad del aire es un tema a considerar" y, de ellos, "un $83 \%$ dice que es un problema muy importante". Estos datos son similares a los encontrados en este estudio, donde el 94,2 \% de la población cree que la contaminación del aire tiene implicaciones en su salud y cuanto mayor nivel formativo tienen, más fuerte es esta asociación. Sin embargo, cuando se pregunta sobre el conjunto de problemas que le preocupan, los aspectos ambientales ocupan un lugar más discreto con el 4,7 \%. Los datos del Barómetro del Centro de Investigaciones Sociológicas (CIS), de diciembre de 2018, indican que el nivel de preocupación de los españoles sobre el medio ambiente es muy bajo, solo lo perciben como tal el 0,1 $\%$ de la población (15). En el ecobarómetro de la Junta de Andalucía del año 2013, que es el último publicado, la calidad del aire ocupa el séptimo lugar entre los problemas más importantes con el 12,7 \%; el primer lugar lo ocupa la suciedad de las calles con el 28,2 \% (17).

En la ciudad de Madrid, municipio con importantes problemas de contaminación, se reconoce, desde el año 2009, que es uno de los principales problemas percibidos, estando entre las 3 primeras causas de preocupación desde el año 2016. En 2017, la contaminación del aire suponía el segundo problema más importante para los madrileños, siendo el primero para los ciudadanos de entre 18 y 44 años y el segundo para el resto (18). En 2019, sigue siendo la segunda causa de preocupación, pero el porcentaje aumenta ligeramente entre los encuestados (de un 31,6 \% a un $32,8 \%$ ) (19).

La opinión de los encuestados en relación a los grupos más vulnerables está en sintonía con lo que indica la literatura científica: los niños, los ancianos y las personas con enfermedades respiratorias son las más afectadas, aunque, en mayor o menor medida, afecta a todas las personas.
Esta percepción resulta similar a la de otros estudios (20).

Por otro lado, los ciudadanos de la Comunidad de Madrid están peor informados que la media de la Unión Europea, donde entre el 20 y el $30 \%$ de los encuestados, según países, dice tener suficiente información (13).

La mayoría de la población estudiada deseaba contar con más información, lo que resulta coherente dada su percepción del riesgo, aunque llama la atención el hecho de que solo un $14,1 \%$ se informa de las superaciones a través de los dispositivos que tienen las Administraciones. Vista la actual normativa sobre calidad del aire y la obligatoriedad de la Administración de informar a los ciudadanos, se necesitará repensar los actuales sistemas para mejorarlos. En este sentido, la Unión Europea ha establecido un modelo basado en colores que sin duda ayudará a mejorar la información que recibe el ciudadano (21).

Recientemente se han publicado varios artículos españoles que relacionan las concentraciones de $\mathrm{NO}_{2}$, PM10 y $\mathrm{O}_{3}$ con la mortalidad de la población. En los mismos se encuentra que Madrid y Barcelona son las ciudades donde esta asociación es más fuerte. También se pone de manifiesto que el aumento en la mortalidad por enfermedades respiratorias y cardiovasculares es una de las consecuencias de la alta concentración de estos contaminantes (22-24). Además, según ciertos autores, esto se asocia con el aumento de prescripciones de medicamentos que tienen como fin controlar estas enfermedades: en el Reino Unido se encontró que un aumento de $10 \mu \mathrm{g} / \mathrm{m}^{3}$ en las PM10, se asocia significativamente con un aumento del $1 \%$ de las prescripciones de salbutamol (23).

Algunos autores apuntan que este hecho debería tener implicaciones en el abordaje terapéutico de los pacientes. Una acción concreta y abordable sería la identificación de individuos en riesgo (25). Debido al infradiagnóstico en enfermedades como la EPOC, se han puesto en marcha varias estrategias para solventar este problema, como el programa de cribado de EPOC en farmacias realizado en Barcelona (26). Pero quizá se puede ir a más, valorando la incorporación de la información de la calidad del aire en los protocolos de atención al paciente alérgico/asmático elaborados por la
Sociedad Española de Farmacia Familiar y Comunitaria (SEFAC) junto con otras sociedades científicas médicas.

En este sentido, abrir la opción a que la farmacia comunitaria pueda informar al paciente asmático, con EPOC y con el conjunto de enfermedades muy vinculadas a la calidad del aire, sería una oportunidad, dado que la red de farmacias en la Comunidad de Madrid alcanza el total de 2.861 establecimientos, de las cuales el $63,4 \%$ se encuentran en la capital. Además, Madrid dispone de la segunda mejor ratio de farmacéuticos por farmacia de toda España, 2,9, 0,6 puntos por encima de la media nacional (27). La farmacia comunitaria se presenta, por tanto, como una red de profesionales muy cualificados en la que, en el marco de los protocolos de atención farmacéutica a los pacientes con enfermedades respiratorias, se podría integrar la información de la calidad del aire de las ciudades. En el caso de la Comunidad de Madrid, esta información es elaborada desde la Dirección General de Salud Pública, siendo remitida a los servicios asistenciales. Podría hacerse llegar también a las farmacias comunitarias, que serían las que informarían al paciente de cuándo la calidad del aire es especialmente perniciosa para su salud. Es una propuesta sobre la que no se ha encontrado experiencia ni en otros países ni en la literatura científica, pero se ve reforzada con la propia opinión que manifiestan los farmacéuticos en el Barómetro de la Farmacia Comunitaria Española (28).

Este trabajo presenta una serie de limitaciones y sesgos entre los que merece la pena destacar que se hizo en la propia farmacia y por los farmacéuticos de la misma, lo que puede inducir un sesgo en las respuestas. Por otro lado, las farmacias rurales estuvieron infrarrepresentadas, aunque los niveles de contaminación en estas áreas son muy bajos.

Como conclusión, la población de la Comunidad de Madrid percibe que no recibe suficiente información sobre cómo la contaminación afecta a su salud y que la farmacia comunitaria podría ser un buen canal para difundir dicha información a los pacientes. Además, se puede integrar en la práctica de la Atención Farmacéutica dados los protocolos y programas articulados disponibles desde SEFAC. 


\section{Agradecimientos}

Los autores agradecen a las doctoras Noelia Tejedor y Mercedes González, de SEFAC y del Colegio Oficial de Farmacéuticos de Madrid (COFM) respectivamente, su colaboración con este estudio y su implicación en el desarrollo. Del mismo modo a la Facultad de Ciencias Experimentales de la Universidad Francisco de Vitoria por la orientación y la ayuda para poder presentar estos resultados. Por último, a las 20 farmacias comunitarias que colaboraron en el estudio y, en especial, a la farmacia de Cristina del Campo Campos donde se pilotó este estudio.

\section{Referencias bibliográficas}

1. Elson D. La contaminación atmosférica. Cátedra. Madrid, 1990

2. Ballester F, Sáez M, Daponte A, Ordóñez JM, Taracido M, Cambra K, et al. El proyecto EMECAS: Protocolo del estudio multicéntrico en España de los efectos a corto plazo de la contaminación atmosférica sobre la salud. Revista Española de Salud Pública. 2005:229-42. https://doi.org/10.1590/ S1135-57272005000200010

3. Di Q; DaiL,Wang Y, Zanobetti A, Choirat C, Schwartz JD, Dominici F. Association of Short-term Exposure to Air Pollution With Mortality in Older Adults. JAMA, 2017;318(24):2446-55. https://doi.org/ 10.1001/jama.2017. 17923

4. López-Campos JL, Márquez-Martín E, Soriano JB. The role of air pollution in COPD and implications for therapy. Expert Rev Respir Med. 2016;10(8):84959. https://doi.org/10.1080/17476348. 2016. 1191356

5. Ordóñez JM, Galán I, Aránguez E. Contaminación atmosférica y efectos en salud de la ciudad de Madrid. Resultados del estudio multicéntrico de efectos de la contaminación atmosférica en salud (Proyecto EMECAS). Madrid: Instituto de Salud Pública; 2005. [acceso 20 de septiembre de 2019]. Disponible en: http://www.madrid.org/ bvirtual/BVCM009573.pdf

6. Guarnieri M, Balmes JR. Outdoor air pollution and asthma. Lancet. 2014;383(9928):1581-92. https://doi. org/10.1016/S0140-6736(14)60617-6

7. Argacha JF, Collart P, Wauters A, Kayaert P, Lochy S, Schoors D, et al. Air pollution and ST-elevation myocardial infarction: A case-crossover study of the Belgian STEMI registry 2009-2013. Int J Cardiol. 2016;223:300-5. https:// doi.org/10.1016/j.ijcard.2016.07.191

8. IARC. Outdoor air pollution a leading environmental cause of cancer deaths. [acceso 20 de septiembre de 2019]. Disponible en https://www.iarc.fr/wp-content/uploads/2018/07/pr221_E.pdf

9. Boletín de la Comunidad de Madrid. Decreto 140/2017, de 21 de noviembre, del Consejo de Gobierno, por el que se aprueba el protocolo marco de actuación durante episodios de alta contaminación por dióxido de nitrógeno $\left(\mathrm{NO}_{2}\right)$ en la Comunidad de Madrid. BOCM, número 279, de 23 de noviembre de 2017.

10. Boletín Oficial del Ayuntamiento de Madrid. Decreto de 15 de enero de 2019 de la Delegada del Área de Gobierno de Medio Ambiente y Movilidad por el que se adoptan en la ciudad de Madrid las medidas de restricción del tráfico y del estacionamiento previstas en el protocolo de actuación para episodios de contaminación por dióxido de nitrógeno en la ciudad de Madrid, aprobado por Acuerdo de la Junta de Gobierno de 10 de diciembre de 2018. BOAM número 8.317, de 17 de enero de 2019.

11. Ministerio de Sanidad y Consumo. Consenso sobre Atención Farmacéutica. MSC. Madrid, 2002.

12. Consejo General de Colegios Farmacéuticos. Código de Deontología de la Profesión 2018. [acceso 20 de septiembre de 2019]. Disponible en: http://www.portalfarma.com/Profesionales/organizacionfcolegial/Documents/2018-Codigo-Deontologia-Profesion-Farmaceutica-CGCOF.pdf

13. Sistema de Vigilancia de la Calidad del Aire del Ayuntamiento de Madrid [Internet]. Mambiente.munimadrid.es. 2020 [acceso 27 de enero de 2020]. Disponible en: http://www.mambiente. munimadrid.es/sica/scripts/index.php

14. Madrid.org - Comunidad de Madrid [Internet]. Gestiona.madrid.org. 2020 [acceso 27 de enero de 2020. Disponible en: http://gestiona.madrid.org/ azul_internet/html/web/DatosEstacion24Accion.icm?ESTADO_MENU=2_1

15. European Comission - Environment. (2019). TNO report. [online] [acceso 2 de junio de 2019]. Disponible en: http://ec.europa.eu/environment/air/ pdf/Survey_AQD_review_PartI_Mainresults.pdf

16. CIS. Barómetro de diciembre de 2018. CIS. [acceso 6 de junio de 2019]. Disponible en: http://www.cis.es/cis/export/sites/default/-Archivos/Marginales/3220_3239/3234/es3234mar.pdf

17. Red de InformaciónAmbiental deAndalucía. Ecobarómetro de Andalucía 2013. Informe de síntesis. [acceso 6 de junio de 2019]. Disponible en: http:// www.juntadeandalucia.es/medioambiente/portal_web/servicios_generales/doc_tecnicos/2013/ecobarometro_2013_sintesis/sintesis2013html/ files/assets/basic-html/index.html\#3

18. Madrid.es. (2017). Encuesta de Calidad de Vida y Satisfacción con los Servicios Públicos de la Ciudad de Madrid -Edición 2017. [online].[acceso 2 de junio de 2019]. Disponible en: https://www.madrid.es/portales/ munimadrid/es/Inicio/El-Ayuntamiento/Calidad-y-Evaluacion/Percepcion-Ciudadana/Edicion-2017/?vgnextfm- $\mathrm{t}=$ defaultctvgnextoid=ba643225968b1610VgnVCM2000001f4a900aRCRDetvgnextchannel $=$ f22ff49c4495d310VgnVCM2000000c205a0aRCRD

19. Madrid.es. (2019). Encuesta de Calidad de Vida y Satisfacción con los Servicios Públicos de la Ciudad de Madrid - Edición 2019. [online]. Àcceso 2 de junio de 2019]. Disponible en: https://www.madrid.es/portales/ munimadrid/es/Inicio/El-Ayuntamiento/Calidad-y-Evaluacion/Percepcion-Ciudadana/Edicion-2019/?vgnextfmt=defaulttrgnextoid=7d062d7c2bd6a610VgnVCM2000001f4a900aRCRDAtvgnextchannel=f22ff49c4495d310VgnVCM2000000c205a0aRCRD

20. Guan WJ, Zheng XY, Chung KF, Zhong NS. Impact of air pollution on the burden of chronic respiratory diseases in China: time for urgent action. Lancet. 2016;388(10054):1939-51. https://doi. org/10.1016/S0140-6736(16)31597-5

21. Airindex.eea.europa.eu. (2019). European Air Quality Index. [online]. [acceso 2 de junio de 2019]. Disponibl en: http://airindex.eea.europa.eu/

22. Ortiz C, Linares C, Carmona R, Diaz J. Evaluation of short-term mortality attributable to particulate matter pollution in Spain. Environ Pollut. 2017;224(224):541-51. https://doi.org/10.1016/j.envpol.2017.02.037

23. Sofianopoulou E, Rushton SP, Diggle PJ, Pless-Mulloli T. Association between respiratory prescribing, air pollution and deprivation, in primary health care. J Public Health (Oxf). 2013;35(4):502-9. https://doi. org/10.1093/pubmed/fdt107

24. Díaz J, Ortiz C, Falcón I, Salvador C, Linares C. Short-term effect of tropospheric ozone on daily mortality in Spain. Atmospheric Environment. 2018;187(187):107-16. https://doi.org/10.1016/j.atmosenv.2018.05.059

25. Cortijo Jimeno J, Morcillo Sánchez EJ. Introducción a la Enfermedad Pulmonar Obstructiva Crónica. España: Consejo General de Colegios Oficiales de Farmacéuticos; 2008.

26. Ortí Juan C, Coval Y. El cribado de EPOC en la oficina de farmacia. El farmacéutico joven [Internet]. 2017 [cited 27 January 2020]; Available from: https://elfarmaceutico.es/index. php/revista-el-farmaceutico-el-farmaceutico-joven/item/8473-el-cribado-de-epoc-en-la-oficina-de-farmacia\#.Xi6r92hKhPY

27. Estadísticas de Colegiados y Farmacias Comunitarias 2017 [Internet]. Elglobal.es. 2017 [cited 2 June 2019]. Available from: https://elglobal.es/ farmacia/el-ano-2017-cierra-con-22046-farmacias-y-suma-78-mas-queel-ano-anterior-da1516270/

28.-Grupo de Investigación en Atención Farncáeutica de la Univerrsdiad de Granada. Barómetro de la Farmacia Comunitaria Española 2016. Edita: Unidad Editorial Revistas. Madrid, 2017. 


\section{Anexo}

Farmacia y Calidad del Aire

\section{Farmacia y Calidad del Aire}

¡Buenos días! Desde el departamento de Salud Pública de la Universidad Francisco de Vitoria nos gustaría que nos respondiese a una serie de preguntas. Nos encontramos estudiando cómo desde la Oficina de Farmacia se puede dar información en materia de Salud Ambiental hacia los pacientes. En concreto queremos ver la demanda que existe de información desde el punto de vista de la contaminación atmosférica de la Comunidad de Madrid, como de los cambios palinológicos que puedan afectar a asma o a alergias.

Sepa que los datos aquí recogidos tienen una finalidad únicamente académica y de investigación, y que un adelanto se presentó ya en el Congreso Nacional de la Sociedad Española de Farmacia Familiar y Comunitaria causando una gran expectación. Se mantendrá en todo momento el anonimato, buscando en encontrar un beneficio final para el paciente. La realización de este cuestionario lleva implícito aceptar el consentimiento informado. Muchas gracias por su tiempo.

*Obligatorio

\section{Dirección de correo electrónico *}

\section{Edad}

Marca solo un óvalo.

$18-40$

$41-65$

Más de 65

\section{Sexo}

Marca solo un óvalo.

Mujer

Hombre

\section{Nivel de estudios}

Marca solo un óvalo.

Sin estudios o con EGB/primaria incompleta

Graduado escolar o primaria completa

ESO, BUP, formación profesional de primer grado

Bachillerato, $\mathrm{COU}$, formación profesional de grado superior

Universitarios

5. ¿Dónde reside? Indique código postal.

6. ¿Dónde trabaja? Indique código postal. 
Farmacia y Calidad del Aire

7. ¿Padece alguna de estas enfermedades? Seleccione una o varias opciones.

Selecciona todos los que correspondan.

\section{Asma}

Bronquitis o EPOC

Hipertensión

Alergia al polen

Enfermedad cardíaca (arritmias, palpitaciones, ha sufrido infartos o anginas de pecho).

No padezco ninguna de las enfermedades anteriores

8. ¿Sabe cuándo se superan los niveles umbral de contaminación en el municipio donde vive?

Marca solo un óvalo.

Sí. A través de los medios de comunicación.

Sí. A través del teléfono móvil.

Sí. A través de comunicaciones institucionales (carteles, viales...)

No

\section{9. ¿Cree usted que la contaminación tiene implicaciones en su salud?} Marca solo un óvalo.
$\bigcirc$ Sí
No
No sé

10. ¿Cree usted que la contaminación de Madrid o del municipio en el que vive le afecta a la salud?

Marca solo un óvalo.

Sí

No

No sé, ni vivo ni trabajo en Madrid

11. ¿Cree que recibe suficiente información sobre las implicaciones de la contaminación en su salud? *

Marca solo un óvalo.

Sí

No

No sé 


\section{Farmacia y Calidad del Aire}

12. ¿A quién cree que afecta más la contaminación? Seleccione las opciones que se vean más afectadas.

Selecciona todos los que correspondan.

\section{Niños}

Adultos sanos

Ancianos

Personas con enfermedades respiratorias (ASMA, EPOC)

Personas con enfermedades cardiacas

Embarazadas

13. ¿Le gustaría recibir información de los efectos de la contaminación en la salud? Marca solo un óvalo.

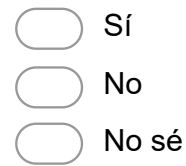

14. ¿Le gustaría que esa información fuera destinada de forma individual a usted? Marca solo un óvalo.

Sí

No

No sé

15. ¿Le gustaría que en la farmacia a la que acude le dieran dicha información relativa a la contaminación de forma individual?

Marca solo un óvalo.

Sí

No

No sé

16. ¿Sabe cuándo hay cambios en los niveles de polen en el municipio donde vive? Marca solo un óvalo.

Sí. A través del sistema de salud (ambulatorios, hospitales, farmacia...)

Sí. A través del teléfono móvil (redes sociales, aplicaciones...)

No

17. ¿Le gustaría recibir información de los cambios en los niveles de polen? Marca solo un óvalo.

Sí

No

No sé 
Farmacia y Calidad del Aire

18. ¿Le gustaría que en la Farmacia a la que acude le dieran dicha información relativa al polen de forma individual?

Marca solo un óvalo.

Sí

No

No sé

Con la tecnología de

: $=$ Google Forms 